\title{
SPATIO-TEMPORAL LOCALIZATION OF AGRICULTURAL MACHINERY OPERATIONS
}

\author{
Dragan Vidaković1, \\ Vuk Malbaša ${ }^{1}$, \\ Zora Konjović2, \\ Endre Pap ${ }^{2}$, \\ Đorđe Obradović ${ }^{1}$
}

\author{
${ }^{1}$ Faculty of Technical Sciences, \\ University of Novi Sad, \\ Novi Sad, Serbia \\ ${ }^{2}$ School of Engineering, \\ University Singidunum, \\ Belgrade, Serbia
}

Correspondence:

Zora Konjović

e-mail:

zkonjovic@singidunum.ac.rs

\begin{abstract}
:
The utilization of agricultural mechanization is crucial to its efficient exploitation that, in turn, significantly influences the entire agricultural economy as well as soil quality. Contemporary agricultural machines are GPS enabled, allowing localization and recording of their motion. In this paper we present an approach to spatio-temporal localization of agricultural mechanization operations based on a linear classifier in fuzzy linear space. We trained a classifier on data labeled using a fuzzy linear classifier in order to create a training set for a data mining predictor that may be helpful in automated management of records describing agricultural machinery's dynamics. The training set was comprised of spatial coordinates of the machine recorded from a GPS device and then manually classified into two classes, denoting whether the machine is utilized for its primary purpose (productive operations like sowing, cultivating, spraying, etc.) or for non-productive operations such as moving the machine in transport from/to fields.
\end{abstract}

Keywords:

Precise farming, GPS, Linear fuzzy space, Classification.

\section{INTRODUCTION}

Operations of tracking and recording motion of agricultural machinery in precision farming have become a very important part of farm management information systems.

Improvements in the accuracy and affordability of the GPS sensors have led to a wide adoption of this technology. The amount of spatio-temporal data automatically collected from these sources increases exponentially. Those data, in the form of time series, need to be transformed into a more useful form (at the operation level) and thereafter may be easy aggregated into a more complex form (at the task level). Agricultural vehicles are not used only for productive operations such as sowing, cultivating, spraying but also for transporting and position changing between two main operations. The main operations are mostly linear, while transporting and position changing are not.

Research in vehicle tracking, agriculture operations recording and precision farming has gained attention of both commercial and scientific communities. For example, companies like Trimble and TopCon, which are known for developing positioning devices, also develop farm monitoring solutions. 
These positioning tools are designed to help farmers in everyday tasks. Services have also been developed to collect data from mechanization, process it into suitable form and present it to the end users (farmers). However, existing tools are expensive and not available to most farmers. On the other hand, a significant amount of research was done on path tracing from GPS data, forming a solid base for the development of sophisticate yet inexpensive devices.

This paper presents an approach to spatio-temporal localization of agricultural mechanization operations based on a linear classifier in fuzzy linear space. The rest of this paper consists of four sections. The first section brings an overview of current solutions and approaches applied in developing such solutions. The second section presents methodology and data used in our research. Third section presents experimental results, while fourth section brings concluding remarks and directions for future work.

\section{RELATED WORK}

Research in vehicle tracking, agriculture operations recording and precision farming has gained attention in both the commercial and scientific communities.

Companies like Trimble and TopCon, which are known for developing positioning products. These positioning tools are designed to help farmers in everyday tasks. Services have also been developed to collect row data from mechanization, process them into suitable form and present it to the end users (farmers). However, those tools are expensive and not available to most farmers.

On the other hand, a significant amount of work was done on path tracing from GPS data. The key technique for identifying paths from GPS data is called Principal Curve Analysis [1]. The basic principal curve algorithm has been improved upon in order to capture the temporal aspect. The basic idea is to find a curve running through the middle of the cloud of points.

Cao and Krumm [2] proposed a method for automatically converting raw GPS traces from everyday vehicles into a routable road network. Their method begins by smoothing the raw traces into a coherent set of paths using a novel aggregation technique that pulls together traces that belong to the same road in response to simulated potential energy wells created around each trace. From those paths, they derived a graph of nodes and edges representing the road network. Compared to the raw GPS traces, their algorithm suppresses the noise present in the raw traces, but that noise is not completely eliminated in all areas.

Corbellini, Ferraris and Parvis [3] designed and tested a low-cost localization system for agricultural applica- tions, which determines a tractor position in fields up to $0.5 \mathrm{~km}$ with an uncertainty of about $1 \mathrm{~m}$. They employed several approaches to obtain the tractor position. Their research shows that GPS systems can have accuracy of 2 m maximum, but the models become much more expensive. For those reasons, their final solution avoids using the GPS and uses laser beams since they have several advantages like substantial immunity with respect to electromagnetic interferences and potentially reduced cost.

Bierlaire, Chen and Newman [4] proposed a probabilistic map matching approach that generates a set of potentially true paths, and associates a likelihood score to each of them. Their model calculates the probability that a GPS recording device would have generated a specific sequence of measurements while following the given path. Their results show that the uncertainty in the path increases with the sparsity of the GPS data. That is consistent with intuition that more GPS data brings more information, reducing uncertainty.

Schuessler and Axhausen [5] described a post-processing procedure applied to the most basic GPS raw data: three-dimensional positions and the corresponding timestamps. They cleaned and smoothed data using a Gaussian kernel, determined trips and activities, and segmented those trips into single-mode stages; the transport mode for each of the stages was also identified. Results show that data obtained from that procedure may be used to develop route and destination choice models.

\section{METHODOLOGY AND DATA}

This section presents methodological approach and data utilized in this paper for spatio-temporal localization of agricultural machinery.

\section{Methodology}

The aim of the proposed algorithm is to classify time series of spatial data into productive operations and transport operations. Main operation lines are described by starting and ending points, average speed, maximal speed and total operating time.

In this research we use fuzzy linear space $[6,7]$ for imprecise data modeling relying upon the basic notion of a fuzzy point.

Definition 1. A fuzzy point $\mathrm{P} \in \mathbb{R}^{2}$, denoted by $\tilde{\mathrm{P}}$ is defined by its membership function $\mu_{\mathrm{p}}^{\sim} \in \mathcal{F}^{2}$, where the set $\mathcal{F}^{2}$ contains all membership functions u: $\mathbb{R}^{2} \rightarrow[0,1]$ satisfying the following conditions: 
i) $\left(\forall u \in \mathcal{F}^{2}\right)\left(\exists_{1} P \in \mathbb{R}^{2}\right) u(P)=1$,

ii) $\left(\forall X_{1}, X_{2} \in \mathbb{R}^{2}\right)(\lambda \in[0,1]) u\left(\lambda X_{1}+(1-\lambda) X_{2}\right) \geq \min \left(u\left(X_{1}\right), u\left(X_{2}\right)\right)$,

iii) function $\mathrm{u}$ is upper semi continuous,

$[u]^{\alpha}=\left\{X \mid X \in \mathbb{R}^{2}, u(X) \geq \alpha\right\}$-cut of the function $\mathrm{u}$ is convex.

The point from $\mathbb{R}^{2}$, with membership function $\mu_{\mathrm{p}}^{\sim}(P)=1$, is denoted by $P(P$ is the core of the fuzzy point $\tilde{\mathrm{P}})$, and the membership function of the point $\tilde{\mathrm{P}}$ is denoted by $\tilde{\mu}_{\mathrm{p}}$. By $[P]^{\alpha}$ we denote the $\alpha$-cut of the fuzzy point (this is a set from $\mathbb{R}^{2}$ ).

Definition 2. $\mathbb{R}^{2}$ Linear fuzzy space is the set $\mathcal{H}^{2} \subset \mathcal{F}^{2}$ of all functions which, in addition to the properties given in Definition 1, are:

Symmetric against the core $\mathrm{S} \in \mathbb{R}^{2}$ :

$(\mu(S)=1), \mu(V)=\mu(M) \wedge \mu(M) \neq 0 \rightarrow d(S, V)=d(S, M)$,

where $d(S, M)$ is the distance in $\mathbb{R}^{2}$.

Inverse-linear decreasing w.r.t. points' distance from the core according to:

$$
\begin{aligned}
& \text { If } r \neq 0, \quad \mu_{\tilde{S}}(V)=\max \left(0,1-\frac{\mathrm{d}(\mathrm{S}, V)}{\left|\mathrm{r}_{\mathrm{S}}\right|}\right) \\
& \text { If } r=0, \mu_{\tilde{S}}(V)= \begin{cases}1 & \text { if } S=V \\
0 & \text { if } S \neq V\end{cases}
\end{aligned}
$$

where $d(S, V)$ is the distance between the point $V$ and the core $S\left(V, S \in R^{n}\right)$ and $\mathrm{r} \in \mathbb{R}$ is constant.

Elements of that space are represented as ordered pairs $\tilde{S}=\left(S, r_{S}\right)$ where $S \in \mathbb{R}^{2}$ is the core of $\tilde{S}$, and $r_{S} \in \mathbb{R}$ is the distance from the core for which the function value becomes 0 .

For the classification task we use two well-known classifiers: logistic regression [8] and artificial neural network [9]. First one is simpler and faster, but less accurate than second one.

Logistic regression is based around the linear modeling of logarithm of the odds ratio of the probability of the positive class and negative class for an example in the training set. It is useful in classification because it explicitly models the class probabilities in the following way:

$$
p\left(y_{i} \mid \mathbf{x}_{i}, \mathbf{r}\right)=\sigma\left(\mathbf{r}^{T} \mathbf{x}_{i}\right)=\frac{1}{1+\exp \left(-\mathbf{r}^{T} \mathbf{x}_{i}\right)}
$$

where $x_{i}$ represents the attributes of an example, $y_{i}$ is the label and $r$ represents the weights of the logistic regression.

The posterior probability may then be expressed as

$$
\begin{aligned}
\mathbf{r}_{\mathrm{MAP}} & =\arg \max _{\mathbf{r}} P(\mathbf{r} \mid D) P(\mathbf{r}) \\
& =\arg \max _{\mathbf{r}} l_{D}(\mathbf{r})+l_{P}(\mathbf{r})
\end{aligned}
$$

Here, in order to compute the optimal weights, we don't take into consideration only $l_{D}$, the loss caused by data, but also $l_{p}$ the loss caused by regularization, for a maximum a posteriori estimate. The weights $r_{(\mathrm{MAP})}$ may be found using an iterative Newton-Raphson solver:

$$
\mathbf{r}^{\text {new }}=\mathbf{r}^{\text {old }}-\eta \mathbf{H}^{-1} \mathbf{g}
$$

Here, the Hessian may be expressed as $\mathbf{H}=\mathbf{X}^{\mathrm{T}} \mathbf{R X}-\mathbf{A}$ where $\mathrm{R}$ is a diagonal matrix with the following elements $R i i=\hat{y}_{i}\left(1-\hat{y}_{i}\right)$ and $\mathrm{A}$ is the precision matrix used for regularization, in this case also diagonal.

The gradient may be computed as

$g=X^{T}(\hat{y}-y)-A r$

Artificial neural networks (ANN) are a biologically inspired models characterized by learning ability. The goal of training in our case is to adjust the weights in a way that the output predicts the correct label for that data point. The network architecture used in this paper is a simple 10x10 feed-forward neural network (Figure 1). The training was performed using backpropagation algorithm.

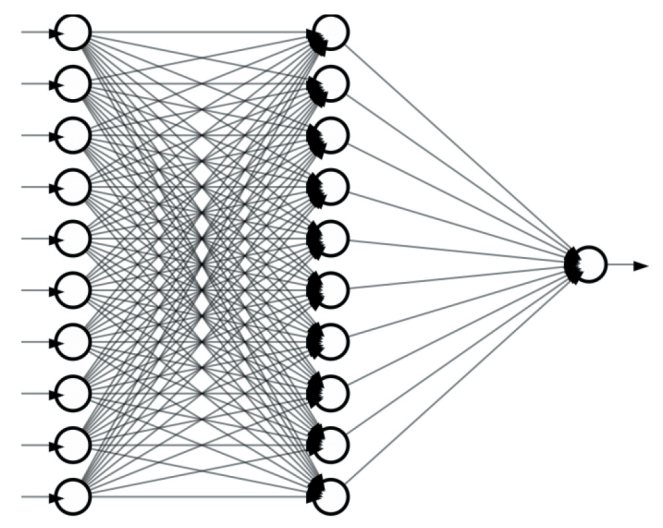

Fig. 1. The ANN architecture

Data

The system for collecting data consists of one GPS sensor equipped with a TCP data communication module. It sends the current position every 2 s to the cloud service. The accuracy of the used GPS sensor is about $1.5 \mathrm{~m}$. The machine was guided by autopilot (second GPS sensor with $1-10 \mathrm{~cm}$ accuracy) along a linear path (during the main operations). The distance between two consecutive main operations should be a multiple of machinery width. 
Annotation of the collected points was done by labeling line segments and curve segments. In this paper we have annotated 401 segments with totally 18280 points. All points that belong to the same segment are then annotated into two classes: line and curve.

Next step in data preprocessing is calculation of the distances between 5 consecutive points $\mathrm{A}, \mathrm{B}, \mathrm{C}, \mathrm{D}, \mathrm{E}$ (Figure 2.).

Distance $\mathrm{AbC}$ is sum of distances $\mathrm{AB}$ and $\mathrm{AC}$. If distance between $\mathrm{AC}$ is equal to distance $\mathrm{AbC}$ then points $\mathrm{ABC}$ are on straight line. However, collected points are imprecise, it means that if distance $\mathrm{AC}$ is smaller than distance $\mathrm{AbC}$ we can't be sure that $\mathrm{ABC}$ is not on line.

As described in section Methodology, in this paper we use fuzzy points in fuzzy linear space to model imprecise data. Also we use triangle inequality to detect fuzzy lines in 5 fuzzy points segment so we calculate distances: $\mathrm{AbC}$, AC, CdE, CE, AbcdE, AE (Figure 2).

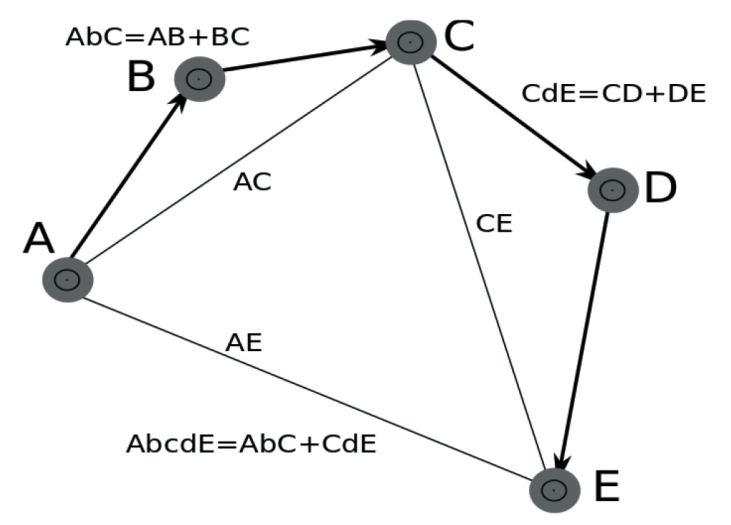

Fig. 2. Distances between 5 consecutive points ABCDE

Finally, after data preprocessing is done, we produce comma separated value file with following columns: $\mathrm{AbC}$, $\mathrm{AC}, \mathrm{CdE}, \mathrm{CE}, \mathrm{AbcdE}, \mathrm{AE}$, speed, cog, lon, lat, segment and class. Columns 0-9 are floats, column 10 is integer (number between 0 and 401) and column 11 is enumeration: 'line', 'curve'. Speed, cog (Course Over Ground), longitude, latitude and segment is related to corresponding attributes of the point $\mathrm{C}$.

\section{EXPERIMENTAL RESULTS}

The data obtained was obtained from sensors mounted on mechanization. It was then pre-processed and labeled according to the described filters. We then trained two machine learning algorithms to predict the label, logis- tic regression and artificial neural networks. $90 \%$ of the examples in the data belong to the positive class as the mechanization is mostly in use while it is moving, the rest are negative examples.

We considered the 10x10 hidden layer architecture of the ANN, with 10 inputs to the network and 1 output (Figure 1.).

The inputs for both the logistic regression and neural networks were $\mathrm{AbC}, \mathrm{AbcdE}, \mathrm{AC}, \mathrm{AE}, \mathrm{CdE}, \mathrm{CE}$, orientation, speed and position data. The data was randomly split into two equally sized categories, training and testing. We trained the logistic regression and neural network models using the training set and tested its performance on the test set. The resulting error was considered a good approximation of generalization error. We measure error as the ratio of correctly labeled examples to total examples. The experiment was replicated 100 times and the results are reported.

In Figure 3 we report the resulting accuracy of 100 logistic regression and 100 neural network models on an ROC curve. The y-axis corresponds to accuracy while the $\mathrm{x}$-axis represents the threshold used. As we can see from Figure 3 the neural network outperforms the logistic regression model.

We used the Levenberg-Marquardt training method for the neural network, which significantly reduced the variance of the resulting model.

Part of the raw data is shown on Figure 4a, where dots represent GPS positions, while lines represent paths between two neighbor points.

Figure $4 \mathrm{~b}$ visualizes a geo-spatial distribution of the classified points. On this figure, circles represent points that constitute non-productive motion, while triangles represent points that constitute productive motion.

The obtained results (points on the very top classified as "non-productive" motion, and those that are not on the top classified as "productive" ones) are common sense, because agricultural machinery usually performs maneuvering in this border area of the field. 

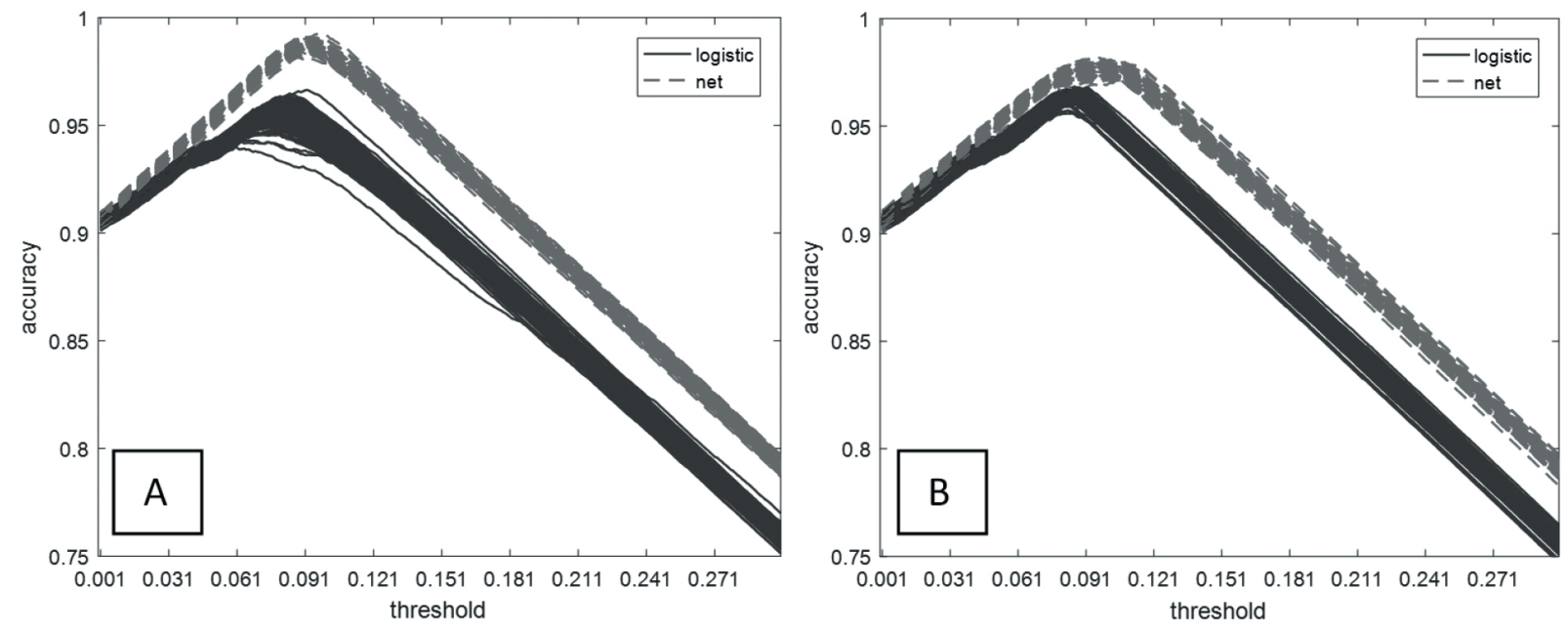

Fig. 3. Linear (logistic) and Neural Network (net) predictor accuracy, using location information A and not B

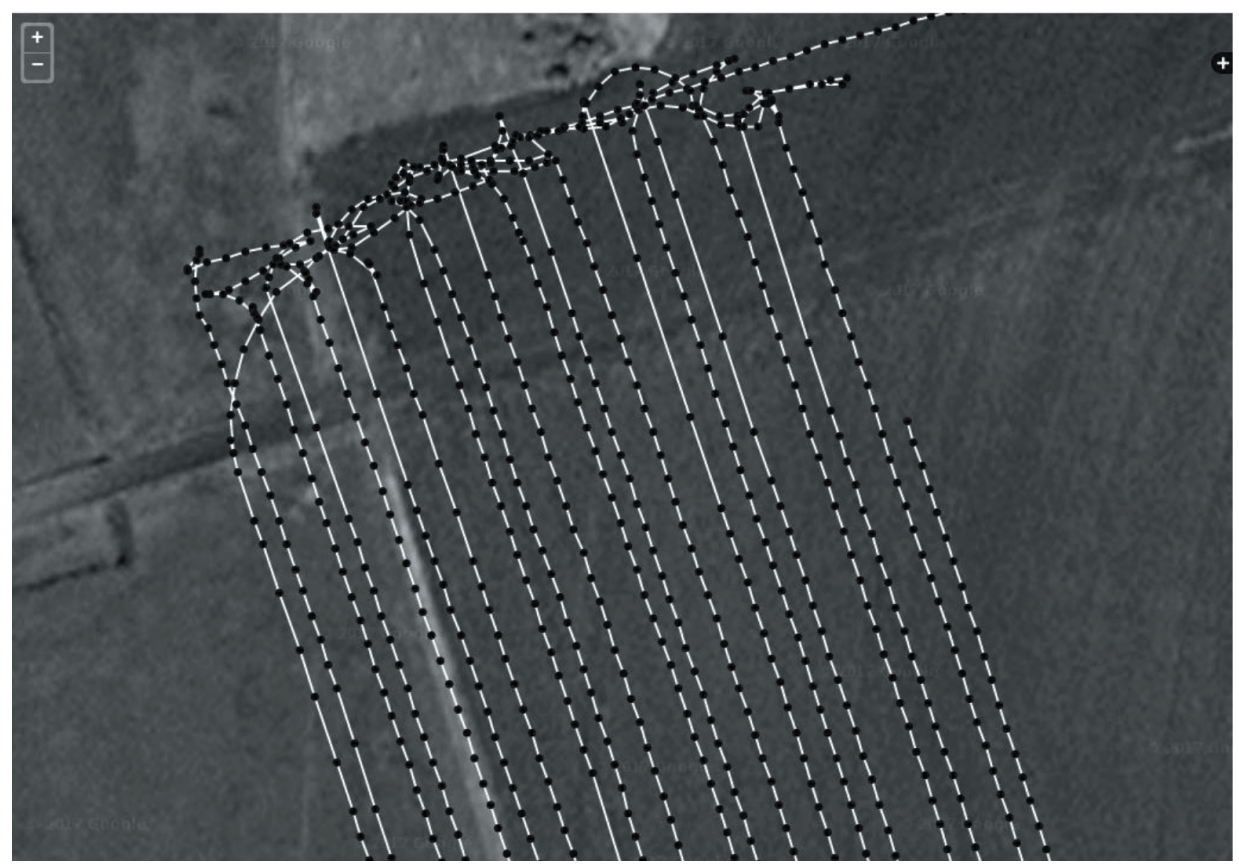

Fig. 4a. Raw GPS data 


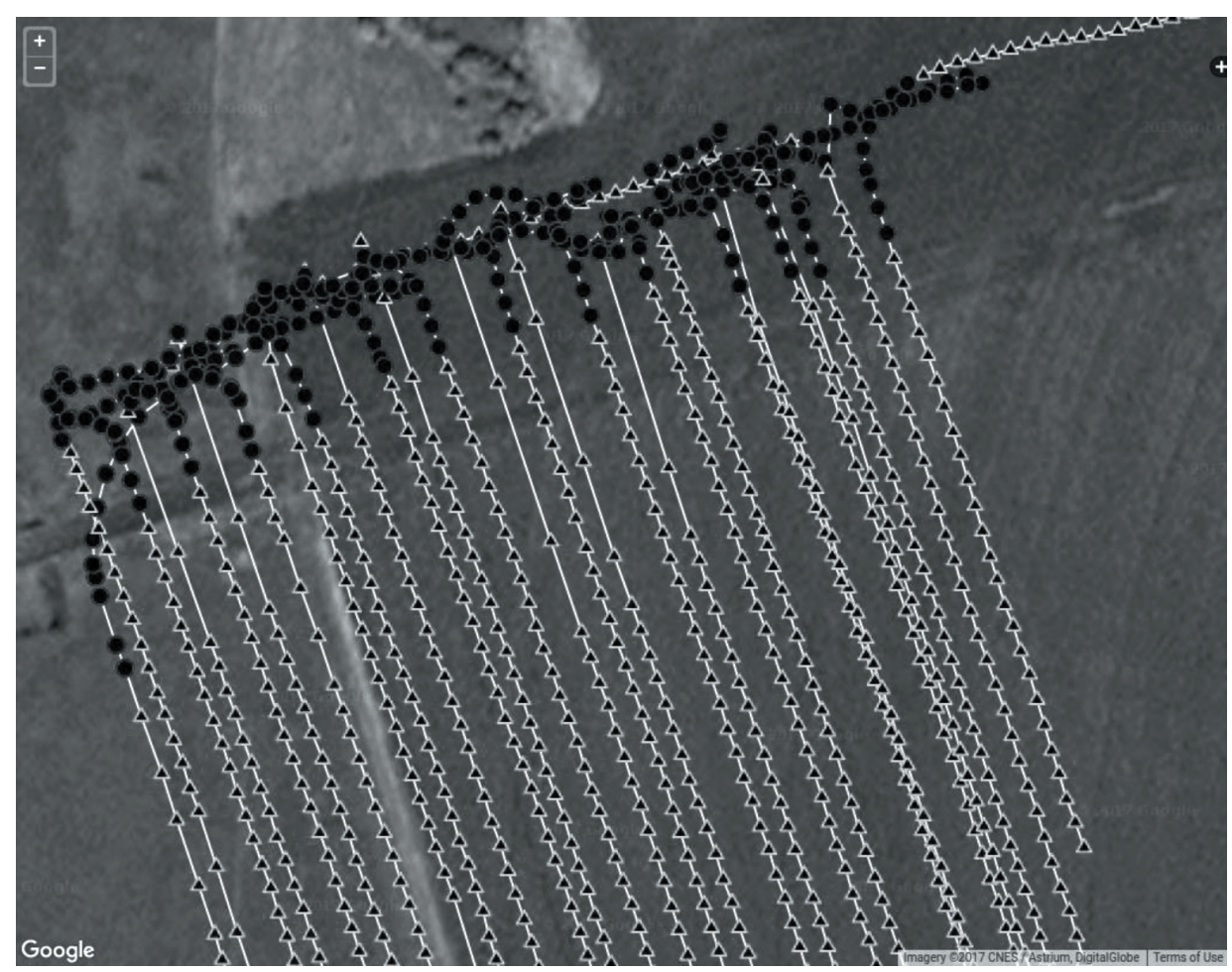

Fig. 4b. An example of classification results

\section{CONCLUSION}

We propose several methods of using machine learning to predict the utilization of mechanization based on measurements which can be gathered directly from mechanization. This is preferable in rural conditions where such mechanization operates.

The results show a significant advantage of the ANN model, as well as lower variance in predictions. The ROC curve shows that the accuracy is a factor of the threshold used, with a slightly more conservative predictor preferred over an accurate class ratio split.

By comparing results visualized on Figures $4 \mathrm{a}$ and $4 \mathrm{~b}$, one can note that the spatial context is an important factor in accuracy.

Future work includes fuzzy line slope detection and correction as well as implementation of a service line (which is usually perpendicular to operations lines) detection algorithm.

Bearing in mind that machine learning techniques underlay the approach proposed in this paper, acquisition of additional data would be desirable as to investigate if and how other factors (for example terrain configuration and alike) influence the final results.

\section{REFERENCES}

[1] T. Hastie, W.Stuetzle, Principal Curves, Journal of the American Statistical Association, Vol. 84, No. 406 (June, 1989), pp. 502-516

[2] L. Cao and J. Krumm, "From GPS traces to a routable road map" in Proceedings of the 17th ACM SIGSPATIAL International Conference on Advances in Geographic Information Systems, pp 3-12, 2009

[3] S. Corbellini, F. Ferraris and M. Parvis, "Low-cost Laser-based Localization System for Agricultural Machines" in Instrumentation and Measurement Technology Conference (IMTC), 2005

[4] M. Bierlaire, J. Chen and J. Newman, "A probabilistic map matching for smartphone GPS data" in Transportation Research Part C: Emerging Technologies, vol. 26., pp 78-98, 2013

[5] N. Schuessler, K. W. Axhausen, "Processing Raw Data from Global Positioning System Without Additional Information" in Transportation Research Record Journal of the Transportation Research Board 2105, pp 28-36, 2009

[6] Đ. Obradović, Z. Konjović, E. Pap, N. M. Ralević, "The maximal distance between imprecise point objects," Fuzzy Sets and Systems, vol. 170, no. 1, pp. 76-94, May. 2011. 
[7] Đ. Obradović, Z. Konjović, E. Pap, I. J. Rudas, "Fuzzy geometry in linear fuzzy space", Intelligent Systems: Models and Applications, pp 137-153, 2013
[8] D. W. Hosmer, Jr., S. Lemeshow, R. X. Sturdivant, Applied Logistic Regression, 3rd Edition, Wiley, 2013.

[9] S. Haykin, Neural Networks and Learning Machines, 3rd Edition, Pearson, 2008. 